\title{
Correction: Impact of COVID-19 lockdown on activity patterns and weight status among youths in China: the COVID-19 Impact on Lifestyle Change Survey (COINLICS)
}

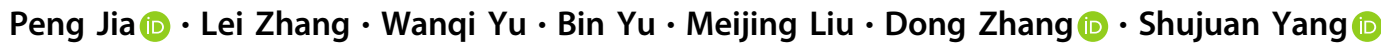

Published online: 12 February 2021

(c) Springer Nature Limited 2021

Correction to: International Journal of Obesity

https://doi.org/10.1038/s41366-020-00710-4

The original version of this article unfortunately contained some mistakes. There is an error detected in the 8th reference: "Jia PA. changed research landscape of youth's obesogenic behaviors and environments in the
post-COVID-19 era. Obesity Rev. 2020. https://doi.org/10. 1111/OBR.13162" should be "Jia P. A changed research landscape of youth's obesogenic behaviors and environments in the post-COVID-19 era. Obesity Rev. 2020. https://doi.org/10.1111/OBR.13162". The original article has been corrected.

There was also an error in the affiliations. 